\title{
Densidad de recurso humano y desigualdad en gastos en salud en países de las Américas
}

\author{
Juan Guerrero Núñez
}

Forma de citar

Guerrero Núñez J. Densidad de recurso humano y desigualdad en gastos en salud en países de las Américas. Rev Panam Salud Publica. 2020;44:e133. https://doi.org/10.26633/RPSP.2020.133

RESUMEN

Objetivo. Determinar existencia de desigualdad y gradiente en gastos de bolsillo, gasto público y gasto privado, entre países americanos estratificados según su densidad de recurso humano en salud (RHS).

Métodos. Estudio analítico y transversal de desigualdades en salud para el gasto de bolsillo (porcentaje del gasto total en salud), el gasto público y el gasto privado (porcentaje del PIB), aplicando la densidad de recurso humano (medicina más enfermería) como estratificador. A partir de datos de la Organización Panamericana de la Salud y el Banco Mundial se categorizaron 32 países americanos según su densidad, se calcularon tasas ponderadas, descriptivos, diferencias, correlaciones, indicadores simples y complejos de desigualdad.

Resultados. Hay alta variabilidad de densidad de RHS (3,8 a 171,3; media de 43,97, DS 37,08) con diferencias significativas entre las categorías de alta y baja densidad. El primer cuartil concentra el $9 \%$ de la población y $4,45 \%$ del RHS; los 3 primeros cuartiles concentran el $48,4 \%$ de la población y el $17 \%$ del RHS. Mediante estratificación, se evidenció un gradiente y desigualdades en indicadores de gastos, mayor en el gasto de bolsillo, con el cual también la densidad de RHS presentó una correlación negativa.

Conclusiones. La densidad de RHS presenta alta variabilidad entre países y grupos categorizados; se concentra en forma desigual en la población, y es mayor en los países con mayor gasto público. Como estratificador de la muestra permitió evidenciar desigualdades y gradientes de gastos en salud; los estratos de menor densidad presentan mayor gasto de bolsillo, menor gasto público y mayor gasto privado.

Palabras clave Evaluación de recursos humanos en salud; gastos en salud; Américas.

Uno de los obstáculos para mejorar el rendimiento y el acceso a la salud ha sido la crisis mundial de la fuerza de trabajo, caracterizada por un déficit y una distribución desigual de los profesionales de salud, especialmente en los países en desarrollo (1). El primer Foro Mundial sobre Recurso Humano en salud (RHS) reconoció la necesidad de enfrentar la crisis y propuso a agentes políticos y de gobierno que se garantice la retención y distribución equitativa de este recurso (2). Para esto, la Organización Mundial de la Salud (OMS) determinó medir la relación entre la población y el RHS mediante el indicador Densidad de Profesionales de Salud, cuya fórmula asociada a la definición de RHS incluye el número de profesionales médicos, enfermeras y parteras, matronas $\mathrm{u}$ obstetrices (3).
En el Informe sobre la salud en el mundo 2006, la OMS señaló un umbral mínimo de densidad de 2,5 profesionales por 1000 habitantes a fin de aumentar las coberturas de atención profesional del parto e inmunizaciones. Otros cálculos proponen un umbral de 2,28 (margen de 2,02 y 2,54) (4). En la Región de las Américas se estableció como meta una densidad de 25 profesionales por 10000 habitantes (3) y se recomendó para el 2015 una densidad de 44,5 por 10 000, considerada esencial para satisfacer las necesidades de salud de la población y alcanzar los Objetivos de Desarrollo Sostenible (5).

En su Informe de 2008 sobre Atención Primaria, la OMS declaró que los RHS son el insumo indispensable para las reformas de la atención primaria y la cobertura universal de salud; sin embargo, muchas personas se veían privadas de atención

\footnotetext{
${ }^{1}$ Universidad de Atacama, Atacama, Chile. $\bowtie$ juan.guerrero@uda.cl
} 
ya que debían financiar gastos médicos de su propio bolsillo, situación que afectaba a 5600 millones de personas en el mundo (6). La falta de acceso a prestadores de atención es una de las principales causas de la inequidad sanitaria (7). La inequidad en salud es un concepto normativo, que puede medirse indirectamente mediante la identificación de las desigualdades en salud, es decir, diferencias observables entre grupos sociales (8). Estas desigualdades son producto de mecanismos estructurales que dan forma a jerarquías sociales de grupos poblacionales y en ningún caso deben considerarse un fenómeno natural (9), corresponden a cualquier variabilidad en aspectos medibles en salud -ya sea en personas o grupos sociales- (10) y se asocian a distintas variables que se manifiestan mediante un gradiente social (11). El estudio de desigualdades requiere metodologías para las cuales existen orientaciones técnicas, como el Manual para el Monitoreo de las desigualdades en salud (8) y la Guía paso a paso para el cálculo de métricas de desigualdad en salud (12). Entre las desigualdades comentadas, la distribución de recurso humano a nivel geográfico o en los distintos niveles de atención es otra evidencia de inequidad (13) que afecta el acceso universal.

En el Informe sobre la salud en el mundo 2010 y financiación de Sistemas de Salud, la OMS señala que por falta de acceso universal 150 millones de personas sufrieron catástrofes financieras y 100 millones fueron obligadas a vivir bajo el umbral de pobreza; en algunos países el 11\% de la población sufría gastos catastróficos y 5\% gastos empobrecedores (14). Se entiende por catástrofe financiera un gasto directo en atención sanitaria que supera el $40 \%$ de los ingresos familiares, deducidos los gastos de alimentación. Este gasto no se debe necesariamente a atenciones de alto costo; puede estar ocasionado por flujos permanentes de atenciones requeridas debido a enfermedades crónicas o discapacidad (14). En algunos países, como Australia, basta con superar el 10\% del ingreso familiar (15). Cuando el pago directo es inferior al 15 a $20 \%$ de los gastos sanitarios totales, la incidencia de desastre financiero es insignificante (14).

El gasto de bolsillo en salud (GBS) se relaciona a diversas variables. Por ejemplo, los pacientes hipertensos de zonas rurales presentan GBS más altos que los de zonas urbanas (16). Otras variables asociadas al aumento del GBS son la hospitalización, ser mujer, el alto nivel socioeconómico y el tamaño familiar (17); sin embargo, los hogares de mayores ingresos gastan más en atenciones pero tienen mayor probabilidad de recibir atención, ser evaluados por médico y recibir medicamentos que los hogares pobres (18). En Perú, 6 de cada 10 adultos mayores presentan GBS, y la localización urbana, el mayor nivel de educación, las enfermedades crónicas y los mayores gastos per cápita son variables que incrementaron hasta 1,6 veces su probabilidad, pero los afiliados al Seguro Integral de Salud, redujeron su GBS en USD 19,3 (19).

La afiliación a seguros de salud contribuye a disminuir el GBS. En México, se redujo en los adultos mayores afiliados a la seguridad social que son atendidos en hospitales públicos (20); en Perú, la afiliación al sistema de salud permitió disminuirlo en los hogares pobres de zonas rurales, asociado al uso exclusivo de servicios entregados por prestadores del primer nivel de atención (21). El seguro nacional de salud de Taiwán, orientado a mitigar la desigual distribución de recursos de atención y cobertura médica, disminuyó el GBS a un 23,08\% en hogares de diferentes regiones e ingresos; sin embargo, la disminución fue desigual y benefició más a los hogares de más recursos (22).
En China, el seguro redujo significativamente la probabilidad de GBS para servicios ambulatorios y de hospitalización, pero aumentó por automedicación (23).

Entre el año 2000 y el 2016, se observó una disminución del GBS de $56 \%$ a $44 \%$ en casi todas las regiones del mundo, explicado mayoritariamente por el aumento del gasto público en salud (24). Sin embargo, en algunos países ha aumentado debido a copagos, honorarios de atención y costos de medicamentos, lo que ha representado una eventual causa de privación de la atención médica o del aumento del abandono del tratamiento (25). Los medicamentos representan la mayor parte del GBS, llegando al 85\% (26). En un estudio de Paraguay en familias con mayores de 60 años enfermos o accidentados se reportó que en familias no pobres y pobres los medicamentos representan el $53,3 \%$ y $71,3 \%$ del GBS, respectivamente, lo que en promedio equivale a 19 días de alimentación y enfrenta a las personas al dilema de comprar alimentos o recibir tratamiento (27).

Por tanto, la crisis de RHS y la inexistencia de seguros de salud, sea por inversión pública o privada, impacta directamente en las personas y familias, poniendo en riesgo sus patrimonios y disminuyendo sus posibilidades de bienestar y mejor salud. Se hace relevante aprender de la experiencia internacional y visualizar cómo se enfrenta la escasez de RHS y su desigual distribución (28).

Considerando que la falta de proveedores de salud es una de las principales causas de inequidad y dado su impacto en los gastos en salud (familiar y sectores público y privado), el objetivo de presente estudio fue determinar la existencia de desigualdad y el gradiente en los gastos de bolsillo, el gasto público y el gasto privado en países de las Américas estratificados según la densidad del recurso humano en salud.

\section{MATERIALES Y MÉTODOS}

Estudio analítico y transversal de desigualdades en salud, con países americanos como unidad de análisis. Los datos de densidad de RHS e indicadores de gastos se obtuvieron de la publicación Indicadores Básicos 2019 de la Organización Panamericana de la Salud (29) y de la Plataforma de información en salud para las Américas (30); las poblaciones se tomaron de la plataforma del Banco Mundial (31). Los análisis se realizaron mediante Excel y SPSS versión 26.

Se construyó y organizó la base de datos según tres dimensiones:

- Indicadores de salud: GBS (\% del gasto total en salud), gasto público y gasto privado (ambos en \% del PIB). Variables dependientes en que se midió desigualdades y gradientes.

- Estratificador de equidad: densidad de RHS (tasa por 10000 habitantes). Variable social con la que se ordenó la distribución de países, sus poblaciones, datos de gastos y a partir de la cual se categorizan grupos. Identifica grupos poblacionales o sociales aventajados y no aventajados.

- Variables demográficas: población de cada país, que permitió construir ponderaciones que brindan mayor o menor peso en los cálculos según la magnitud poblacional.

La muestra correspondió a 32 países con datos completos, ordenada de menor a mayor densidad de RHS. El enfoque metodológico general se basó en: 1) generar una escala de estratos según cuartiles, basados en la densidad de RHS, 2) analizar 
la distribución de densidad e indicadores de gastos, y 3) aplicar métricas de desigualdad simples y complejas.

1) La escala de estratos se categorizó según cuartiles de densidad de RHS en alta, media alta, media baja y baja. Para cada categoría, se calculó la tasa ponderada de densidad de RHS mediante la sumatoria de las ponderaciones de densidad de RHS, calculadas en base a la población de cada país respecto al total poblacional de cada categoría. El mismo cálculo ponderado se aplicó para obtener tasas ponderadas de indicadores de gastos para cada categoría.

2) Se calcularon descriptivos de tendencia central y dispersión (desviación estándar y coeficiente de variación). Dado que mediante la prueba de Shapiro Wilk se descartó una distribución normal de densidad de RHS y de uno de los indicadores de gastos, se aplicaron pruebas no paramétricas (coeficiente de Spearman para evaluar la asociación entre densidad de RHS e indicadores de gastos y de
Kruskal-Wallis con prueba post hoc [prueba por parejas] para evaluar diferencias entre categorías de densidad, con nivel de significancia del 0,05 ).

3) Con las tasas ponderadas de densidad de RHS y de los 3 indicadores (gastos) se calcularon métricas simples de desigualdad: brecha absoluta, equivalente a la diferencia aritmética entre categorías extremas (q4-q1) y brecha relativa, mediante la razón de estas (q4/q1). Para obtener métricas complejas se empleó la guía técnica (12) y se calculó: índice de desigualdad de la pendiente (IDP) mediante cálculo de regresión de tasas que permite determinar gradiente absoluto (mediante Excel e indicaciones de la Guía citada), donde los resultados negativos equivalen a mayor indicador de gasto en estratos vulnerables (baja densidad de RHS) y los resultados positivos lo contrario; índice de concentración de la desigualdad en salud (ICS) que permite determinar el gradiente relativo, mostrando el grado en que se concentra el indicador, cuyo valor al ser negativo indica concentración en desmedro de estratos

CUADRO 1. Países de las Américas y gastos en salud, categorizados según la densidad de recursos humanos para la salud, 2019

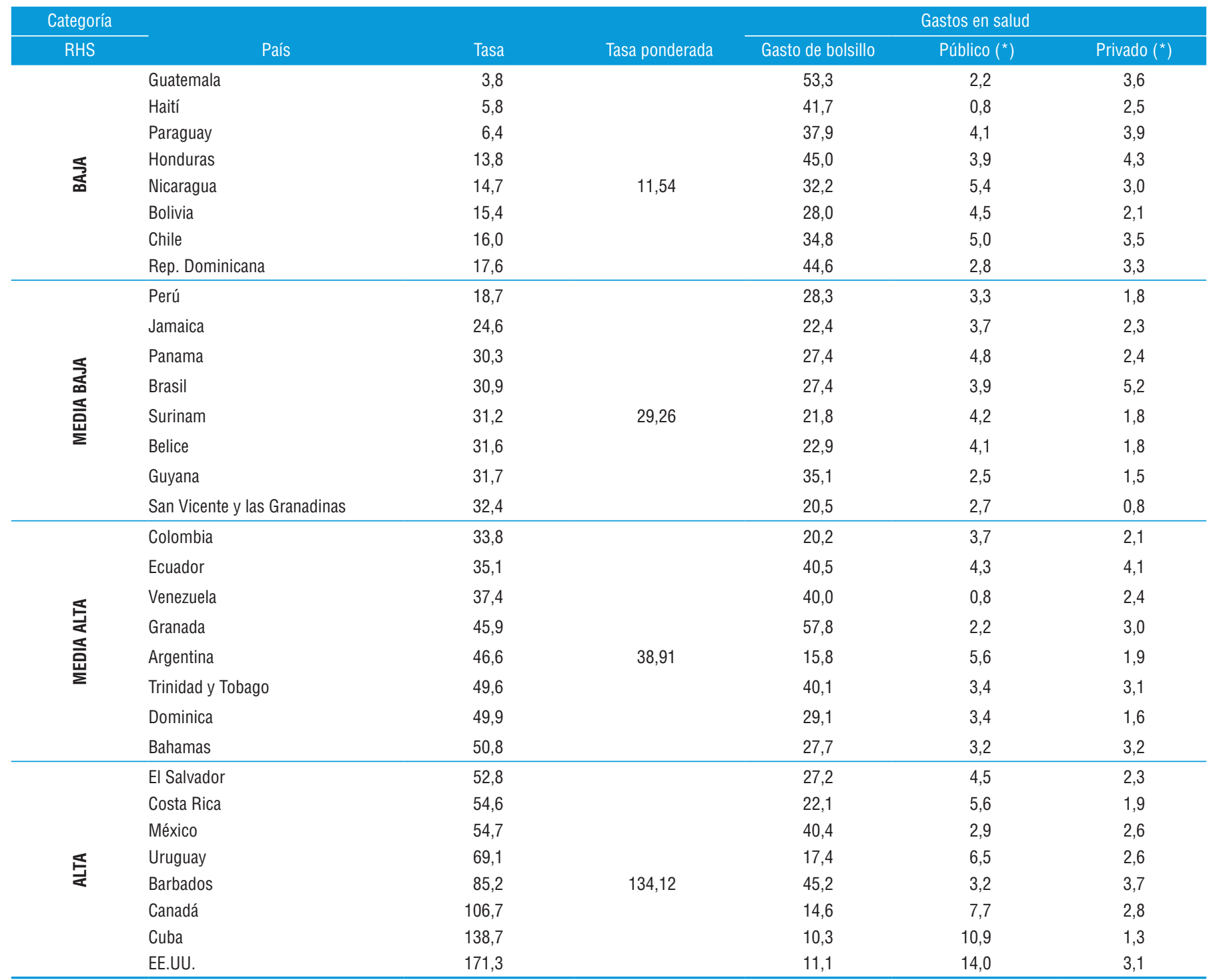

*Gasto público y privado, expresado como porcentaje del producto interno bruto (PIB).

Fuente: Elaboración propia, a partir de datos de Publicación Indicadores Básicos 2019, OPS. 
desaventajados, considerando que valores de 0,2 o 0,3 representan un nivel alto de desigualdad. Esta se complementa con la curva de concentración, que cuando se encuentra bajo la línea de igualdad indica concentración en grupos aventajados, y lo contrario cuando se ubica sobre ella.

Dado el tipo de estudio, que los datos utilizados son de acceso público y no incluyen datos sensibles o personales, no se necesitó evaluación de un Comité de Ética.

\section{RESULTADOS}

La densidad de RHS presenta una distribución heterogénea, de alta variabilidad, desde 3,8 (Guatemala) hasta 171,3 (EE.UU.), con una razón entre ambos de 45, una media de 43,97, un desvío estándar de 37,08 y un coeficiente de variación de 0,84, mayor en las categorías extremas (0,48 en alta, 0,46 en baja y 0,16 en medias). En las categorías alta y baja (Cuadro 1), se observa que países de mayor y menor densidad, difieren en 3,24 (EE.UU.-El Salvador) y 4,6 veces (República Dominicana-Guatemala). Existen 19 países con densidad inferior a la media y 10 bajo el umbral crítico (equivalente a 25). La prueba de Kruskal-Wallis $(29,091 ; p<0,001)$, con una significancia de 0,05 y prueba posthoc (prueba por parejas) demostró diferencias significativas entre grupos de categorías: baja-alta, baja-media alta, alta-media baja. Mediante el coeficiente de correlación de Spearman solo se comprobó una correlación (negativa) con el indicador GBS, con una significancia de 0,05 (Spearman -0,442; $p=0,011$ ).

Respecto de las métricas de desigualdad en densidad de RHS se determinó una brecha absoluta de 123 y una brecha relativa de 12 , lo que evidencia que el estrato desaventajado (baja) posee 123 profesionales menos por 10000 habitantes, equivalente a 12 veces menos que el estrato más aventajado. Presenta un índice de desigualdad de la pendiente de 195 con error típico de 31,54 y un índice de concentración de $-0,33$. La curva de concentración (Figura 1) muestra que el 9\%, 34\% y $48 \%$ de la población, concentra el $1 \%, 10 \%$ y $17 \%$ de densidad de RHS, respectivamente.

Con respecto a los indicadores de gastos, a partir de los valores ponderados según población para cada categoría se observaron desigualdades en su proporción (Figura 2) y sus respectivas métricas (Cuadro 2). El GBS presenta 14 países sobre la media $(30,71 \%)$, de los cuales 7 pertenecen a la categoría baja, 5 a las medias y 2 a la alta. Los 3 países de mayor densidad de RHS (EE.UU., 171,3; Cuba, 138,7; y Canadá, 106,7) presentan los GBS más bajos (11\%, 10\% y $14 \%$, respectivamente). El país de menor densidad (Guatemala, 3,8) presenta el segundo GBS más alto $(53,3 \%)$. Presenta un gradiente, desde menor gasto en la categoría de alta densidad a mayor gasto en la de baja densidad, con brecha absoluta y relativa positivas y superiores a los otros dos tipos de gastos; con un índice de desigualdad de la pendiente negativo, que confirma gradiente con afectación de categoría más vulnerable (baja densidad) e índice de concentración, que no manifiesta un nivel alto de concentración.

Con respecto al gasto público, 11 países se ubican sobre la media $(4,37 \%), 3$ de los cuales están en la categoría baja, 2 en las medias y 6 en la alta, con un mayor gasto en el grupo de países de alta densidad. Presenta alta variabilidad, desde $0,8 \%$ (Haití) hasta 14\% (EE.UU.), con una razón entre ambos de 17,5 veces. Los tres países de mayor densidad (EE.UU., Cuba y Canadá) poseen los gastos más altos (14\%, 10,9\% y 7,7\%,
FIGURA 1. Desigualdad relativa de densidad de recursos humanos en salud, presentada en curva de concentración, para 32 países americanos, 2019

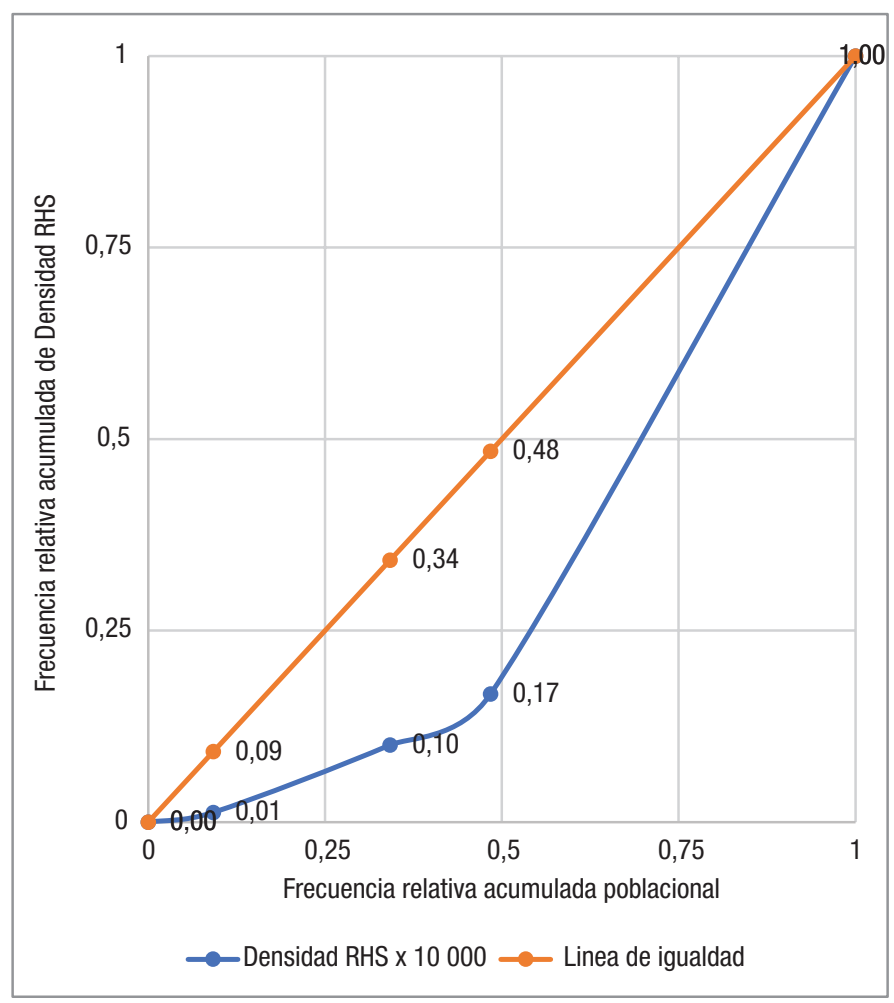

Fuente: Elaboración propia a partir de datos de Publicación Indicadores Básicos 2019, OPS y datos Banco Mundial.

FIGURA 2. Proporción y tasa de gastos ponderados, estratificados según densidad de recursos humanos en salud, para 32 países americanos, 2019

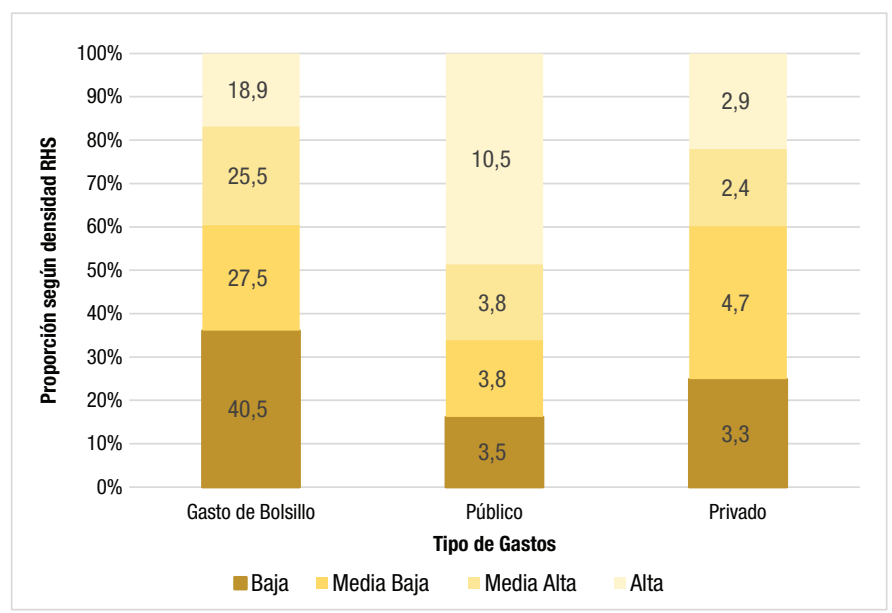

Unidades: Gasto de Bolsillo (\% de Gasto total en salud), Gasto Público y Privado (\% PIB) Fuente: Elaboración propia a partir de datos de Publicación Indicadores Básicos 2019, OPS y datos Banco Mundial.

respectivamente). Presenta asimismo un gradiente, con mayor gasto en el grupo de países categorizados como de alta densidad. Las diferencias absolutas negativas y relativas menores a cero dan cuenta del menor gasto representado por el cuartil 1. 
CUADRO 2. Desigualdad en gastos en salud, según estratificación por densidad de recursos humanos en salud en países americanos, 2019

\begin{tabular}{|c|c|c|c|c|c|c|c|c|}
\hline \multirow[b]{2}{*}{ Tipos de gastos } & \multicolumn{4}{|c|}{ CATEGORÍAS } & \multicolumn{2}{|c|}{ BRECHA } & \multirow{2}{*}{$\begin{array}{l}\text { ÍNDICE DE LA } \\
\text { PENDIENTE }\end{array}$} & \multirow{2}{*}{$\begin{array}{c}\text { INDICE DE } \\
\text { CONCENTRACIÓN }\end{array}$} \\
\hline & Baja Cuartil 1 & Media baja Cuartil 2 & Media alta Cuartil 3 & Alta Cuartil 4 & Absoluta (q1-q4) & Relativa (q1/q4) & & \\
\hline Gastos de bolsillo & 40,50 & 27,46 & 25,54 & 18,86 & 21,64 & 2,15 & $-22,69$ & 0,13 \\
\hline Gasto público & 3,50 & 3,83 & 3,77 & 10,51 & $-7,01$ & 0,33 & 12,08 & $-0,23$ \\
\hline Gasto privado & 3,28 & 4,66 & 2,35 & 2,89 & 0,39 & 1,13 & $-1,91$ & 0,08 \\
\hline
\end{tabular}

Fuente: Elaboración propia a partir de datos de Publicación Indicadores Básicos 2019, OPS y datos Banco Mundial.

FIGURA 3. Desigualdad relativa de gastos en salud según estratificación por densidad de recursos humanos en salud, representada en curva de concentración, para 32 países americanos, 2019

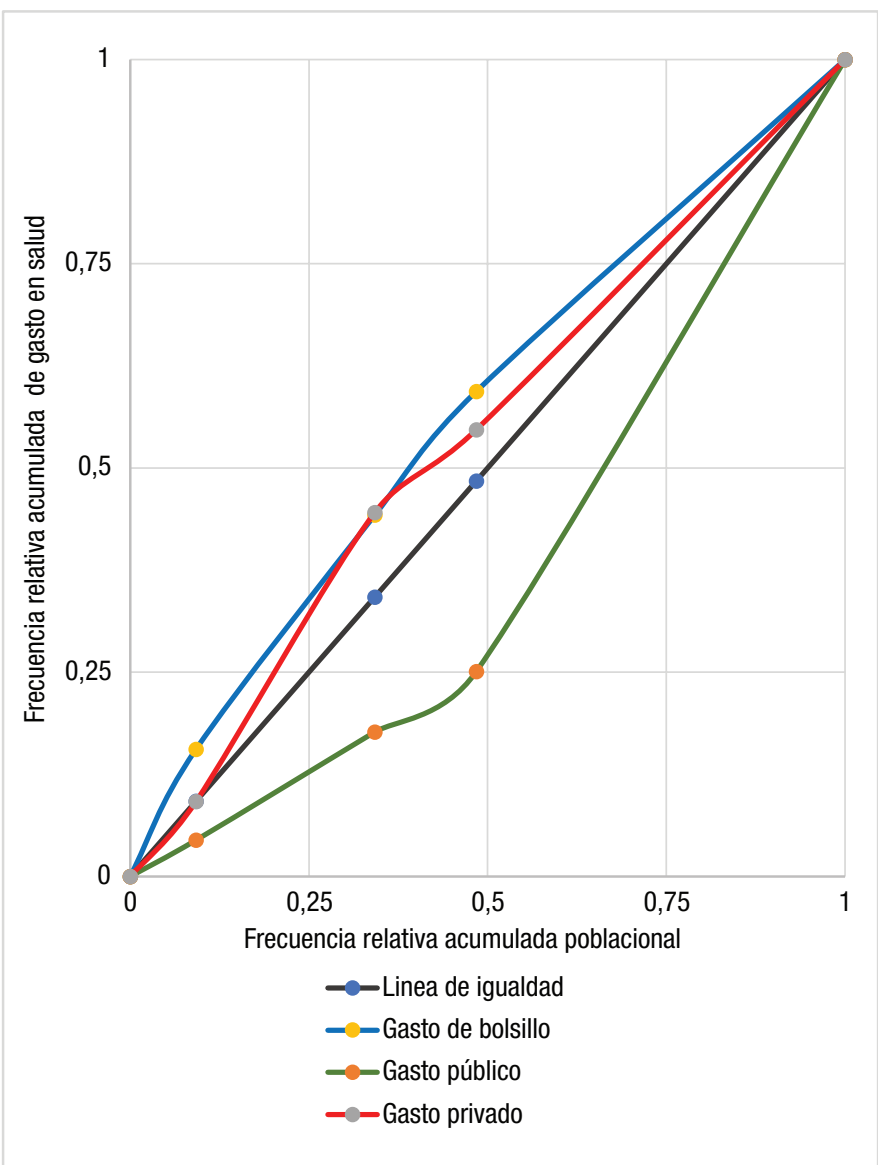

Fuente: Elaboración propia a partir de datos de Publicación Indicadores Básicos 2019, OPS y datos Banco Mundial.

Presenta un índice de la pendiente positiva de 12 puntos y un índice de concentración considerado de nivel alto.

El gasto privado presentó una media de 2,67\%, con 14 países por encima de ella y una diferencia de 6,5 veces entre el país con el mayor indicador (Brasil) y aquel con el menor indicador (San Vicente y las Granadinas). Aunque según sus medias ponderadas para cada categoría (Figura 2 y Cuadro 2) no se observa claramente un efecto de gradiente, se debe considerar que es un indicador de menor intervalo y variabilidad. Pese a esto, presenta una mayor brecha relativa que el gasto público y un índice de la pendiente negativo, equivalente al $41 \%$ del mayor valor (categoría media baja). Su índice de concentración es menor al de los otros indicadores.

La curva de concentración (Figura 3) muestra una mayor concentración en el gasto público, y es la única curva ubicada por debajo de la diagonal, lo que indica que es mayor en el estrato aventajado. Se observa que el $48 \%$ de la población con menor densidad de RHS concentra 25\% del gasto público, el 59\% del GBS y el 55\% del gasto privado.

\section{DISCUSIÓN}

El análisis cuantitativo de los datos de densidad de RHS actuales y su distribución geográfica en la Región de las Américas demuestra avances importantes para alcanzar la tasa de 25 profesionales por 10000 habitantes, indispensable para las atenciones básicas. Sin embargo, estos son insuficientes frente al umbral recomendado para 2015 de una densidad de 44,5 profesionales por 10000 habitantes, considerada necesaria para el logro de los Objetivos de Desarrollo Sostenible (5). De los 32 países analizados, 10 se ubican debajo del umbral crítico de 25 y 19 debajo del umbral de 44,5, y representan en conjunto más de 438 millones de personas (casi el $44 \%$ de la población americana).

Esta realidad, oculta ante la media de 44, reaparece frente a descriptivos de dispersión y métricas de desigualdad, con alta variabilidad observada y a la vez una alta concentración, evidenciando que el $83 \%$ de la densidad de RHS se concentra en el $52 \%$ de la población (Figura 1). En términos prácticos, esto se traduce en que el $48 \%$ de la población solo dispone del 17\% de los RHS. Además, las diferencias significativas entre estratos permiten afirmar que a las diferencias entre países (45 veces entre EE.UU. y Guatemala) se agrega la de grupos categorizados (Cuadro 1) y hace presumir diferencias al interior de cada país, no solo en diferencias absolutas de RHS sino también en su distribución en las distintas zonas geográficas. Estas diferencias dan cuenta de las desigualdades en la disponibilidad de recursos necesarios para atención básica y para enfrentar los desafíos comprometidos al 2030, entre los que se destacan objetivos en salud maternoinfantil, enfermedades no transmisibles, VIH y otras enfermedades transmisibles de interés regional. Esto no solo afecta los objetivos sanitarios enunciados, sino que además compromete las aspiraciones regionales de lograr una cobertura sanitaria universal y, en particular, la protección contra riesgos financieros.

La aplicación de la densidad de RHS como estratificador permitió evidenciar la existencia de desigualdades, gradientes de gastos y grados de concentración (Figuras 2 y 3), además de una correlación negativa solo con el GBS. Los estratos de menor densidad de RHS poseen mayor GBS, menor gasto público y mayor gasto privado, y desde el enfoque de desigualdades en salud constituyen 
grupos vulnerables y desaventajados respecto de los que tienen mayor disponibilidad de RHS. Entre antecedentes recientes a considerar, Haití posee mayor tasa de mortalidad infantil, con la mayor desigualdad, según la brecha del indicador entre madre de mayor y menor educación y entre quintiles de mayor y menor riqueza; en mortalidad atribuible a todas las causas aparecen con las mayores tasas Haití, Honduras, Guatemala, Nicaragua y Bolivia (32), países categorizados con baja densidad de RHS, mayor GBS y que muestran mayor afectación del estado de salud.

Respecto del gasto público, es importante notar que los tres primeros estratos no presentan gran variabilidad de sus valores $(3,5$ a 3,8\%). Por tanto, las brechas absolutas y relativas, respecto del estrato alta densidad (10,51\%), no serían muy distintas si se comparara con estratos medios. Las desigualdades observadas se basan en la mayor concentración de aportación pública en la mayoría de los países de alta densidad de RHS y que además presentan menores valores de GBS, lo que concuerda con el descenso asociado al aumento del gasto público de otras observaciones (24). Es urgente analizar la necesidad de avanzar hacia una mayor eficiencia del gasto público en salud; datos recientes de la OMS demuestran que, en la Región, este se concentra en actividades curativas y proporcionalmente es más pequeño en países de baja densidad de RHS (p. ej., Haití y Guatemala), mientras que la República Dominicana presenta el menor gasto en atención preventiva. De ahí la necesidad de no solo evaluar la factibilidad de aumentar el gasto público, sino también evitar gastos innecesarios, invertir en áreas de mayor eficiencia de acciones en salud y avanzar hacia una menor dependencia en el gasto privado para lograr una cobertura universal de alta calidad (32). Esto es más importante aún si se considera que, según el presente estudio, el gasto privado muestra un gradiente con ascenso hacia grupos de menor densidad de RHS.

Los agentes políticos y comunitarios, los gestores y los tomadores de decisión deberían considerar las implicancias de estas evidencias. Si bien el estudio las revela a nivel ecológico y no individual, la evidencia existente señala afectación de los individuos y familias frente a procesos de enfermedad asociados a altos índices de gastos propios, que pueden conducir incluso a catástrofe financiera (15). Los gobiernos, al generar reformas e implementar políticas en salud, deberían potenciar no solo las estrategias asociadas a los Objetivos de Desarrollo Sostenible relacionadas con el financiamiento, la contratación, el desarrollo, la capacitación y la retención de personal sanitario, sino también orientar sistemas de financiamiento hacia la disminución del GBS (33) apoyados por investigación para generar políticas pertinentes (34).

Dado el rol de determinante social de la densidad de RHS, se deberá evaluar la evidencia internacional para generar estrategias de mejora. Malawi, Etiopía y Perú, con una correcta planificación de estrategias y recursos, revirtieron la crisis en pocos años; Brasil y Tailandia lo demostraron con voluntad política, inversiones adecuadas y una gestión eficaz a largo plazo; Filipinas demostró que una gran producción de trabajadores no asegura la atención sanitaria en zonas marginadas a menos que existan estrategias eficaces para atraer y mantener el personal en zonas rurales, tal como lo lograron exitosamente Malí y Zambia (28). Guatemala, que presenta la más baja densidad de RHS $(3,8)$ y un $20 \%$ de la población que no cuenta con servicios de salud, ha contado con el apoyo de Cuba para la formación de médicos y el establecimiento de criterios sociales para la asignación de estos a regiones rurales y apartadas (35).

Independientemente de causas estructurales o intermedias que expliquen las desigualdades, el análisis de densidad de RHS y gastos no solo evidencia desigualdad y gradiente sino también la concentración de recursos. Ello representa un obstáculo para disminuir globalmente las inequidades en salud (7), un aspecto aún más relevante frente al escenario actual de pandemia por la COVID-19 y los crecientes desafíos para los RHS.

Este estudio tiene algunas limitaciones. Los datos poblacionales pueden tener variaciones; las interpretaciones deben ser cautelosas ya que se aplican al nivel de la muestra y no a comunidades locales ni individuos.

En conclusión, la densidad de RHS presenta alta variabilidad entre países y grupos categorizados, se concentra en forma desigual entre la población, y es mayor en países con mayor gasto público. Como estratificador de la muestra permitió evidenciar desigualdades y gradientes de gastos en salud; los estratos de menor densidad poseen mayor gasto de bolsillo, menor gasto público y mayor gasto privado.

Los resultados se deben interpretar con moderación; se requiere profundizar con estudios longitudinales que permitan evaluar tendencias de cambios en las desigualdades de la densidad de RHS e indicadores de gastos en salud.

Contribución de los autores. El autor llevó a cabo el diseño y el desarrollo del estudio, y aprobó la versión final aceptada para publicación.

\section{Financiamiento. Ninguno.}

\section{Conflicto de intereses. Ninguno declarado.}

Declaración. Las opiniones expresadas en este manuscrito son únicamente responsabilidad del autor y no reflejan necesariamente las de la Revista Panamericana de Salud Pública o la Organización Panamericana de la Salud.

\section{REFERENCIAS}

1. Dal Poz M. A crise da força de trabalho em saúde. Cad Saúde Pública. 2013; 29(10):1924-1926. Available from: http://www. scielo.br / scielo.php?script=sci_arttext\&pid=S0102-311X201300 1000002\&lng=en. https://doi.org/10.1590/0102-311XPE011013.

2. Organización Mundial de la Salud \& Alianza Mundial en pro del Personal Sanitario. Declaración de Kampala y prioridades para la acción internacional. Ginebra: Organización Mundial de la Salud; 2008. Disponible en: https://apps.who.int/iris/handle $/ 10665 / 77831$
3. Organización Panamericana de la Salud. Manual de Medición y Monitoreo de Indicadores de las metas regionales de recursos humanos para la salud: un compromiso compartido. Washington DC: OPS; 2011. Disponible en: https://www.observatoriorh.org/ manual-de-medicion-y-monitoreo-indicadores-de-las-metas-regionales-de-recursos-humanos-para-la-salud

4. Organización Mundial de la Salud. Informe sobre la salud en el mundo 2006 - Colaboremos por la salud. Ginebra: OMS; 2006. Disponible en: https://www.who.int/whr/2006/es / 
5. Organización Mundial de la Salud. Estrategia de Recursos Humanos para el acceso universal a la salud y la cobertura universal de salud. 29a Sanitaria Panamericana. Washington DC: OPS; 2017. Disponible en: https://www.paho.org/hq/index. php? option $=$ com_docman\&view $=$ download \&category_slu$\mathrm{g}=29$-es-9250\&alias=42334-csp29-r15-s-334\&Itemid=270\&lang=es

6. Organización Mundial de la Salud. La atención primaria de salud más necesaria que nunca. Informe sobre la salud en el mundo 2008. Informe de un Grupo Científico de la OMS; Ginebra: OMS; 2008. Disponible en: https://www.who.int/whr/2008/ 08_report_es.pdf?ua $=1$

7. Organización Mundial de la Salud. Aumentar el acceso al personal sanitario en zonas remotas o rurales mejorando la retención: recomendaciones mundiales de política. Ginebra: OMS; 2011. Disponible en: https://www.who.int/hrh/retention/guidelines/ es/

8. Organización Panamericana de la Salud. Manual para el Monitoreo de las Desigualdades en Salud, con especial énfasis en países de ingresos medianos y bajos. Washington DC: OPS; 2016. Disponible en: https://www.paho.org/hq/index.php?option=com_ content\&view=article\&id=12571: manual-monitoreo-desigualdadessalud-paises-ingresos-medianos-bajos\&Itemid=39529\&lang=en

9. Silva M. Revisión sobre las causas de las desigualdades en salud, incluyendo las condiciones de empleo. Arch Prev Riesgos Labor. 2018;21(3):162-163

10. Arcaya M, Arcaya A, Subramanian S. Desigualdades en Salud: definiciones, conceptos y teorías. Rev Panam Salud Publica. 2015;38(4):261-271.

11. Mújica Ó, Moreno C. From words to action: measuring health inequalities to "leave no one behind". Rev Panam Salud Publica. 2019;43:e12.

12. EWEC-LAC. Guía paso a paso para el cálculo de métricas de desigualdad en salud. Todas las mujeres, todos los niños; 2017. Disponible en: https://www.everywomaneverychild-lac.org/publica tion/guia-calculo-desigualdad-salud/

13. Lucio C, Recaman A, Arredondo A. Evidencias sobre la inequidad en la distribución de recursos humanos en salud. Horizonte sanitario. 2018;17(1):77-82.

14. Organización Mundial de la Salud. Informe sobre la salud en el mundo: la financiación de los sistemas de salud: el camino hacia la cobertura universal. Ginebra: OMS; 2010. Disponible en: https:// www.who.int/whr/2010/es/

15. Callander E, Fox H, Lindsay D. Out-of-pocket healthcare expenditure in Australia: trends, inequalities and the impact on household living standards in a high-income country with a universal health care system. Health Econ Rev. 2019;9(1):10.

16. Baker-Goering M, Roy K, Howard D. Relationship Between Adherence to Antihypertensive Medication Regimen and Out-of-Pocket Costs Among People Aged 35 to 64 With Employer-Sponsored Health Insurance. Prev Chronic Dis. 2019;16:E32.

17. Attia-Konan A, Serge A, Toure A, Kouadio K. Distribution of out of pocket health expenditures in a sub-Saharan Africa country: evidence from the national survey of household standard of living, Cote d'Ivoire. BMC Res Notes. 2019;12(1):25.

18. Makinen M, Waters H, Rauch M, Almagambetova N, Bitran R, Gilson $\mathrm{L}$, et al. Inequalities in health care use and expenditures: empirical data from eight developing countries and countries in transition. Bull World Health Organ. 2000;78(1):55-65.

19. Hernandez A, Rojas C, Santero M, Prado F, Roselli D. [Healthrelated out-of-pocket expenses in older peruvian adults: analysis of the national household survey on living conditions and poverty 2017]. Rev Peru Med Exp Salud Publica. 2018;35(3):390-9.
20. Salinas G, Carrillo M, Pérez M, Garcia C. [Out of pocket expenditure on health during the last year of life of Mexican elderly: analysis of the Enasem]. Salud Publica Mex. 2019;61(4):504-13.

21. Petrera M, Jiménez E. Determinants of out-of-pocket spending on health among the poor population served by public health services in Peru, 2010-2014. Rev Panam Salud Publica. 2018;42:e20.

22. Chu T, Liu T, Chen C, Tsai Y, Chiu W. Household out-of-pocket medical expenditures and National Health Insurance in Taiwan: income and regional inequality. BMC Health Serv Res. 2005;5:60.

23. Du J, Yang X, Chen M, Wang Z. Socioeconomic determinants of out-of-pocket pharmaceutical expenditure among middle-aged and elderly adults based on the China Health and Retirement Longitudinal Survey. BMJ Open. 2019;9(7):e024936.

24. Xu K, Soucat A, Kutzin J, Brindley C, Vande N, Toure H, et al. Public Spending on Health: A Closer Look at Global Trends, O.M.d.l. Salud, Editor. 2018, Organización Mundial de la Salud. Disponible en: https://www.who.int/health_financing/documents/ health-expenditure-report-2018/en/.

25. Yusuf F, Leeder S. Recent estimates of the out-of-pocket expenditure on health care in Australia. Aust Health Rev. 2020;44:340-6.

26. Rasul F, Kalmus O, Sarket M, Adid H, Hossain S, Hasan Z, et al. Determinants of health seeking behavior for chronic non-communicable diseases and related out-of-pocket expenditure: results from a cross-sectional survey in northern Bangladesh. J Health Popul Nutr. 2019;38(1):48.

27. Giménez E, Caballero N, Peralta N, Araujo J. Análisis del gasto de bolsillo ante la enfermedad de personas de 60 y más años según su condición de pobreza en Paraguay. Rev Salud Publica Paraguay. 2019;9:46-52.

28. Dayrit M, Dolea C, Dreesch N. Abordando la crisis de los Recursos Humanos para la Salud: ¿Hasta dónde hemos llegado? ¿Qué podemos esperar lograr al 2015? Rev Per Med Exp Salud Publica. 2011;28(2):327-36.

29. Organización Panamericana de la Salud. Indicadores Básicos 2019. Tendencias de la salud en las Américas. Washington DC: OPS; 2019. Disponible en: https://iris.paho.org/bitstream/ handle /10665.2/51543/9789275321287_spa.pdf? sequence=7\&is Allowed $=\mathrm{y}$

30. Organización Panamericana de la Salud. PLISA: Plataforma de información en salud en las Américas. Washington DC: OPS; 2019. Disponible en: https://www.paho.org/data/index.php/es/

31. Banco Mundial. Plataforma Banco Mundial, sección Datos; 2019. Disponible en: https://datos.bancomundial.org/indicator/SP.POP. TOTL

32. OECD/The World Bank. Panorama de la Salud: Latinoamérica y el Caribe 2020. Paris: OECD Publishing; 2020. Disponible en: https:// doi.org/10.1787/740f9640-es

33. García H, Díaz P, Ávila D, Cuzco M. La Reforma del Sector Salud y los recursos humanos en salud. An Fac Medic. 2015;76:7-26.

34. Fu W, Zhao S, Zhang Y, Chai P, Goss J. Research in health policy making in China: out-of-pocket payments in Healthy China 2030. BMJ. 2018;360:k234.

35. Gonzalez E, Gómez L, López G. Guatemala: paradigma en la formación de recursos humanos en salud. Edumecentro. 2013;5(3):241-8.

Manuscrito recibido el 15 de enero de 2020. Aceptado para su publicación, tras revisión, el 2 de septiembre de 2020 


\section{Human resource density and inequality in health care spending in the Americas}

ABSTRACT Objective. To determine the existence of inequality and gradient in out-of-pocket expenses, public spending and private spending, among countries from the Americas stratified according to their human resources for health $(\mathrm{HRH})$ density.

Methods. Analytical and transversal study of health inequalities for out-of-pocket spending (percentage of total health spending), public spending and private spending (percentage of GDP), applying the human resource density (medicine and nursing) as a stratifier. Based on data from the Pan American Health Organization and the World Bank, 32 countries from the Americas were categorized according to their density, and weighted rates, descriptors, differences, correlations, and simple and complex indicators of inequality were calculated. Results. There is high variability in $\mathrm{HRH}$ density (3.8 to 171.3; mean 43.97, SD 37.08) with significant differences between high and low density categories. The first quartile concentrates $9 \%$ of the population and $4.45 \%$ of the $\mathrm{HRH}$; the first 3 quartiles concentrate $48.4 \%$ of the population and $17 \%$ of the $\mathrm{HRH}$. The stratification showed a gradient and inequalities in expenditure indicators, higher in the out-of-pocket expenditure, with which the $\mathrm{HRH}$ density presented a negative correlation.

Conclusions. HRH density shows high variability among countries and categorized groups; it is unevenly concentrated in the population, and is greater in countries with higher public spending. As a stratifier of the sample, it showed inequalities and gradients in health spending; the strata with lower density showed higher out-of-pocket spending, lower public spending and higher private spending.

Keywords Health human resource evaluation; health expenditures; Americas. 